\title{
LOW MOLECULAR WEIGHT AGENTS SUPPORT SURVIVAL OF CULTURED NEURONS FROM THE CENTRAL NERVOUS SYSTEM
}

\author{
SILVIO VARON, ${ }^{1}$ STEPHEN D. SKAPER, GILLES BARBIN, ${ }^{2}$ IVAN SELAK, ${ }^{3}$ AND \\ MARSTON MANTHORPE
}

Department of Biology, School of Medicine, University of California, San Diego, La Jolla, California 92093

Received April 7, 1983; Revised August 17, 1983; Accepted $\Lambda$ ugust 19, 1983

\begin{abstract}
Microcultures of dissociated neurons from various central and peripheral neural tissues were used for quantitative analysis and characterization of trophic agents directed to these test neurons. Media conditioned by a variety of central and peripheral glial as well as muscle cell cultures contain trophic activities for central neurons which are distinct from the more traditional protein factors directed to peripheral neurons, by at least two features: (1) they reside with low molecular weight $\left(M_{\mathrm{r}}<1000\right)$ agents which are resistant to heat, extremes of $\mathrm{pH}$, and various proteolytic and peptidolytic enzymes; and (2) they are necessary for the short-term survival of a variety of rodent and avian central neurons, but they fail by themselves to support survival of peripheral neurons under the same culture conditions.
\end{abstract}

Neurons in the developing peripheral nervous system (PNS) are known to require protien-like trophic factors for their survival in vitro, and a similar requirement has been shown in vivo at least in some cases (reviewed by Varon and Adler, 1980,1981). Much less clear is whether neurons in the central nervous system (CNS) also depend on macromolecular trophic factors. Several conditioned media (CMs), from glial and muscle cell cultures, contain survival-promoting proteins for PNS neurons (Barde et al., 1978; Helfand et al., 1978; Varon et al., 1981). The same CMs also contain trophic agents for spinal cord neurons which, however, are dialyzable and trypsin-resistant and thus unlikely to be proteins (Longo et al., 1982; Manthorpe et al., 1982). Astrological CM has also been reported to contain trophic activity for hippocampal neurons (Banker, 1980; Müller and Seifert, 1982).

We have recently developed dissociated neuronal cultures suitable for the detection and investigation of trophic agents acting on intrinsic fetal rat CNS neurons, the survival of which is also supported by $\mathrm{CMs}$ from various central and peripheral glial cultures (Barbin et al., 1984). In the present study, we have employed rat

\footnotetext{
1 To whom correspondence should be addressed.

2 Visiting Centre National de la Recherche Scientifique scientist from the Groupe de Neuropharmacologie Biochimique, College de France, Paris (France).

3 Visiting Fonds National de la Recherche Scientifique scientist from the Départment de Clinique et de Pathologie médicale, University of Liége (Belgium).
}

and chick CNS neuronal cultures to examine molecular properties and neuronal target specificities of trophic activities present in various CMs. We report that all of the CNS-directed trophic activities of these CMs reside with low molecular weight $\left(M_{\mathrm{r}}<1000\right)$ agents, while all of their PNS-directed ones associated with higher molecular weight $\left(M_{\mathrm{r}}>10,000\right)$ agents. The trophic agents for CNS neurons are characterized by resistance to heat, acid and alkaline $\mathrm{pHs}$, and several proteases and peptidases. These features and the low molecular size define a new class of trophic agents, the essential requirement for which appears restricted to CNS neurons.

\section{Materials and Methods}

Media and cultures. Media, dissociation procedures, neural cultures, and methodology for trophic bioassays have been described in detail previously (see below). In the present work, the medium used was HEBM (i.e., Eagle's Basal Medium modified to contain $26.4 \mathrm{~mm}$ $\mathrm{NaHCO}_{3}, 33.3 \mathrm{mM}$ D-glucose, $2 \mathrm{mM}$ L-glutamine, and 100 units/ml of penicillin), supplemented as indicated below for PNS and CNS cell cultures. All cultures were fixed with glutaraldehyde after $24 \mathrm{hr}$ of incubation $\left(37^{\circ} \mathrm{C}, 5 \%\right.$ $\mathrm{CO}_{2}$-air), and the number of neurons surviving in each well was calculated from neuronal counts taken under phase microscopy over representative $0.5 \times 6 \mathrm{~mm}$ diametral strips.

PNS test neurons (Longo et al., 1983; Manthorpe et al., 1983) were obtained from neonatal mouse dorsal root 
ganglia (mDRG), embryonic day 10 (E10) chick dorsal root ganglia (cDRG10), E11 chick sympathetic ganglia (cSG11), or E8 chick ciliary ganglia (cCG8). Six-millimeter microwells (Costar no. 3596, 96 microwells/plate) were precoated with polyornithine and polyornithinebinding neurite-promoting factor (Manthorpe et al., 1981), which forms a neurite-permissive substratum and thereby facilitates neuronal recognition without providing neuronotrophic stimulus itself. Polyornithine alone was equally effective. The wells then received $50 \mu \mathrm{l}$ of serially diluted test materials (CMs), or fractions thereof, and $50 \mu \mathrm{l}$ of test neuron suspension $(20,000$ neurons $/ \mathrm{ml})$. The final medium was HEBM supplemented with $10 \%$ fetal calf serum. Hippocampal, striatal, and septal neurons from 18-day fetal rat (Barbin et al., 1984) were obtained by collecting the tissue in $\mathrm{Ca}^{2+}, \mathrm{Mg}^{2+}$-free balanced salt solution, treating with $0.08 \%$ trypsin in the same solution for $30 \mathrm{~min}$ at $37^{\circ} \mathrm{C}$, and dissociating by trituration in serum-free HEBM containing $1 \%(\mathrm{w} / \mathrm{v})$ ovalbumin. The dissociated cells were filtered through a $20-\mu \mathrm{m}$ mesh and diluted to 20,000 cells $/ \mathrm{ml}$ in the final culture medium HEBM- $2 \times$ N1, i.e., serum-free HEBM supplemented with twice the normal concentrations of 5 $\mu \mathrm{g} / \mathrm{ml}$ of insulin, $5 \mu \mathrm{g} / \mathrm{ml}$ of transferrin, $1 \times 10^{-4} \mathrm{M}$ putrescine, $2 \times 10^{-8} \mathrm{M}$ progesterone, and $3 \times 10^{-8}$ $\mathrm{Na}_{2} \mathrm{SeO}_{3}$ (Skaper et al., 1979).

A more convenient source of CNS test neurons than the fetal rat tissues could be provided by 8 -day chick embryo forebrain (cFB8), because of both the routine availability of this tissue and its higher cell yield. We have applied to the cFB8 tissue the dissociation procedure described above for rat CNS and generated cell suspensions with a yield of about $2 \times 10^{7}$ cells/embryo. In previous studies with fetal rat CNS cultures, we had established that neuronal identification for neuronal cell counts could rely on morphological criteria under phase contrast microscopy with a high degree of correlation to tetanus toxin binding assessed by fluorescence microscopy (Barbin et al., 1984). The same analysis was carried out with the new cFB8 cell cultures: nearly all of the cells surviving at $24 \mathrm{hr}$ were identifiable as neurons by the morphological criteria and were verified to be tetanus toxin-binding cells (Fig. 1) - currently the most reliable criterion for neuronal identity in neural cell cultures (Mirsky et al., 1978). As with the fetal rat cultures (Barbin et al., 1984), it was first necessary to verify the cell seeding density which would allow for no neuronal survival in the absence of exogenous trophic support but a maximal percentage of survival in its presence. Figure $2 A$ shows the increasing neuronal survival induced by increasing seeding densities in the absence of CM supplementation, as well as the suitability of seeding densities below 5000 cells/well to provide optimal bioassay conditions for exogenous trophic agents. A typical bioassay curve, using 1000 cells/well, is illustrated in Figure $2 B$ : the dilution achieving half-maximal survival support defines the trophic titer (trophic units (TU) per milliliter) of the material being tested.

Conditioned media and ultrafiltration fractions. Conditioned media (Manthorpe et al., 1982) were obtained from rat $\mathrm{RN} 22$ schwannoma (RCM), rat $\mathrm{C} 6$ glioma (C6CM), purified primary mouse Schwann cells (SCM)
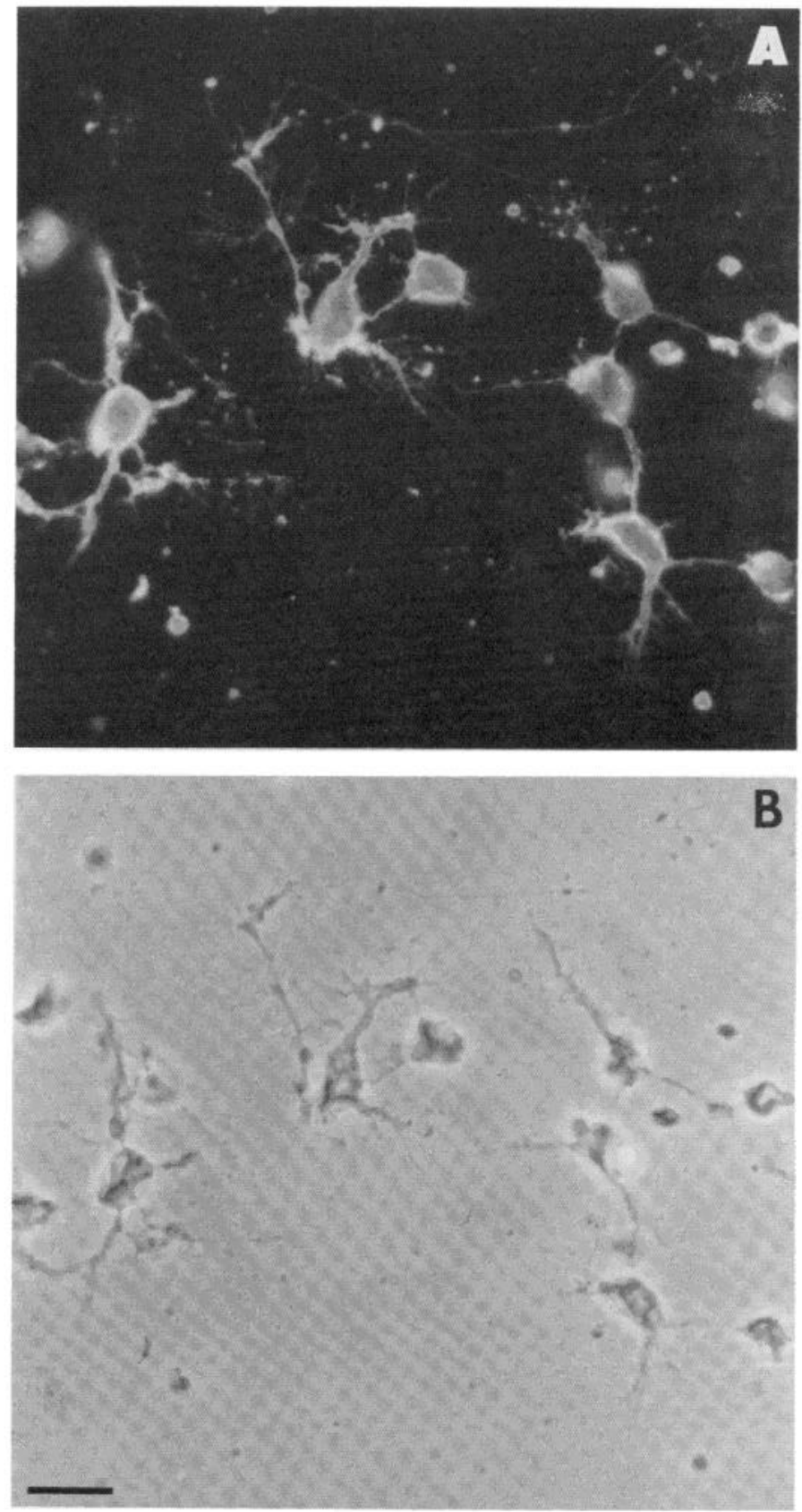

Figure 1. Tetanus toxin labeling of embryonic chick forebrain cultures. Cells were seeded at $10^{5}$ per culture and treated after $24 \mathrm{hr}$ for tetanus toxin binding as described previously (Barbin et al., 1984). Photomicrographs were taken from the same field with an Olympus inverted microscope using Kodak Pan-X film. Exposure times were $1 / 2$ sec for fluorescent $(A)$ and $1 / 8$ sec for phase $(B)$ images. Magnification bar $=50 \mu \mathrm{m}$.

and rat astroglia (ACM), or 8-day chick embryo heart muscle cells (HCM) by exposing serum-free HEBM medium for $48 \mathrm{hr}$ to the corresponding monolayer cell cultures. Crude CMs were applied to an Amicon ultrafiltration PM10 membrane, having a molecular weight cutoff of 10,000 daltons, until $90 \%$ of the loaded volume had filtered through. The filtrates (F10 fractions) were collected directly. The retentates (R10 fractions) were further washed on the PM10 membrane with unconditioned HEBM and finally reconstituted with HEBM to 

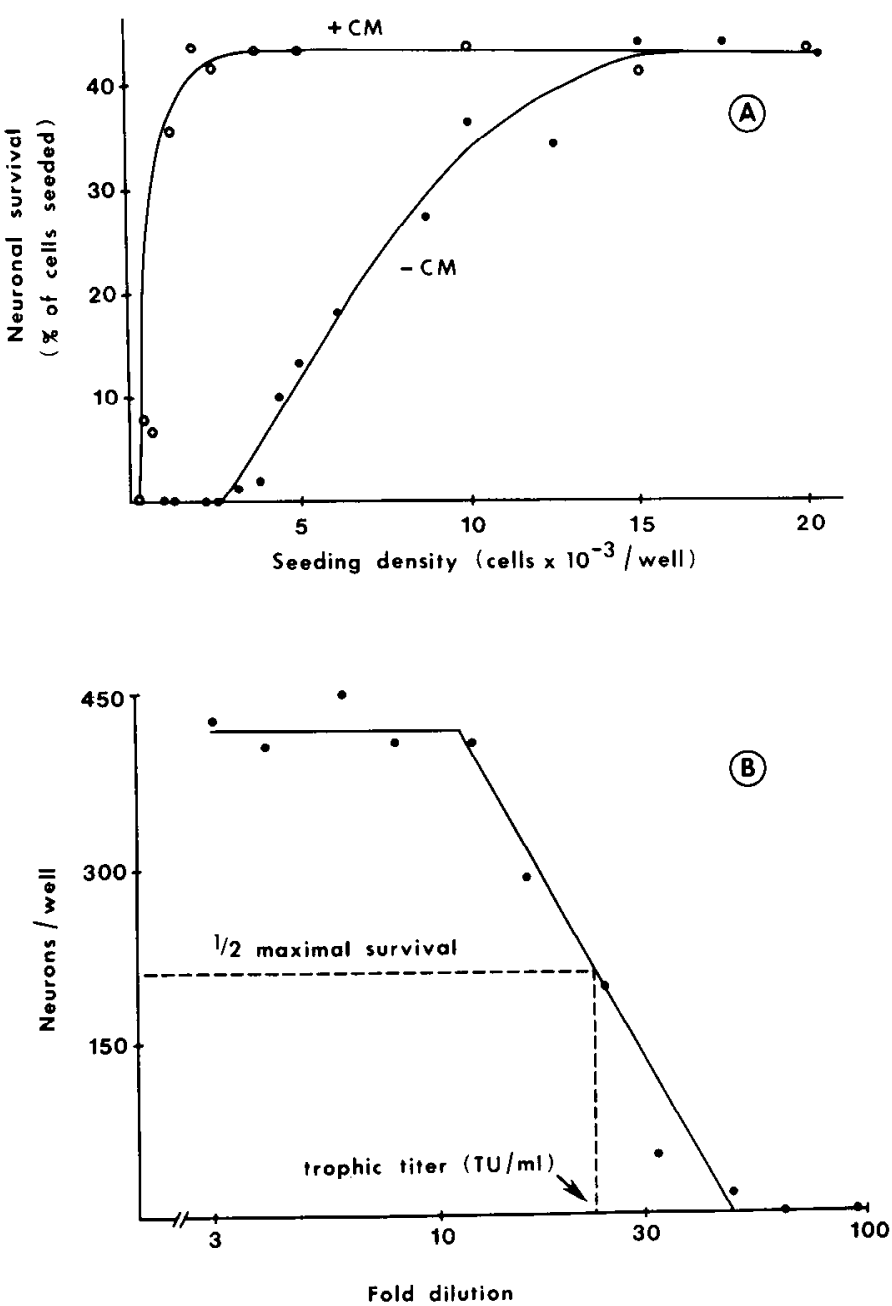

Figure 2. Chick embryo forebrain (cFB8) cell cultures. Dissociated forebrain cells were seeded into 6 -mm wells precoated with polyornithine and polyornithine-binding neurite-promoting factor. The cultures were incubated for $24 \mathrm{hr}$ in serum-free. HEBM-N1 medium in the presence or absence of CM. Surviving neurons were counted in the fixed cultures under phase contrast microscopy. (For details, see "Materials and Methods.") $A$, Density-dependent neuronal survival in the absence $(-)$ or presence $(\mathrm{O}-\mathrm{O})$ of trophic support by CM (1:10). $B$, A low density (1000 cells/well) culture system is used to titrate the trophic activity of serially diluted CM.

the original volume of their crude CM. In some experiments, the F10 fractions were further applied to a UM2 membrane (nominal molecular weight cutoff $=1,000$ daltons), and the corresponding $\mathrm{R} 2$ retentate and F2 filtrate fractions were collected and tested.

\section{Results}

Segregation by ultrafiltration of trophic agents addressing CNS and PNS neurons. The five serum-free CMs selected for investigation were processed through PM10 membranes. Crude CMs and their R10 and F10 fractions were then assayed for trophic activities using in parallel the four CNS and the four PNS neuronal test populations. Table I shows the results.

All five crude CMs displayed trophic activity for all four CNS neuronal cultures. For each CM, the trophic titer was essentially the same regardless of the CNS test neurons used, suggesting a common trophic agent effective on all neuronal targets. CNS trophic titers did vary across different CMs. However, no attempts were made in these studies to match the number of cells in the conditioning cultures or to define for each culture the optimal conditioning paradigm. All crude $\mathrm{CMs}$ also had trophic activities for the PNS test neurons, but at lower levels than for the CNS ones. PNS activities in CMs generated in the absence of serum (see "Materials and Methods") were lower than those previously reported in serum-containing CMs (cf. Adler and Varon, 1980; Varon et al., 1981). Besides variation among different CMs, there were clear differences among the titers expressed by each CM for different PNS test neurons, suggesting either the presence of different trophic molecules addressing different ganglionic cells or a different responsiveness of each neuronal type to the same agent.

The R10 fractions of all CMs displayed no activity for any CNS neuronal population, while retaining practically all of the activities present in the crude CMs for the PNS test neurons. A contrasting behavior was displayed by the F10 filtrates, which had retained completely the trophic activity for CNS neurons but had lost the trophic activities for PNS neurons. Therefore, we could conclude that (1) CNS- and PNS-directed trophic activities of these CMs reside on different molecules which can be fully segregated from one another by PM10 ultrafiltration, and (2) the CNS activity in all CMs and for all CNS test neurons resides with a molecule or molecules with a molecular size lower than 10,000 daltons.

The next approach was to define further the upper limit of molecular size for the CNS-directed trophic agents. When $\mathrm{F} 10$ fractions from the various $\mathrm{CMs}$ were applied to a UM2 ultrafiltration membrane, all of the activity was recovered in the F2 filtrates (Table I, bottom). Thus, the CNS trophic activity of all the CMs examined here must be associated with molecules in the range of 1000 daltons (the cutoff of UM2 membranes) or smaller.

Additional properties of the CNS-directed trophic agents present in RCM. The F10 fraction from RCM was divided into several aliquots. Each aliquot was subjected to a different treatment and was then tested for trophic content using all four CNS neuronal test systems. Separate aliquots were (1) kept for $3 \mathrm{hr}$ at $4^{\circ} \mathrm{C}$ (control); (2) incubated at $100^{\circ} \mathrm{C}$ for $30 \mathrm{~min}$, cooled, and kept at $4^{\circ} \mathrm{C}$ for the rest of the time; (3) adjusted to $\mathrm{pH} 4$ or $\mathrm{pH} 10$, kept on ice for $3 \mathrm{hr}$, and then brought back to $\mathrm{pH} 7$; (4) exposed to enzyme solutions in $1 \mathrm{mM} \mathrm{HCl}$, for a final enzyme concentration of $40 \mu \mathrm{g} / \mathrm{ml}$-specifically, trypsin (3000 units/mg, porcine pancreas, Calbiochem), $\alpha$-chymotrypsin (1140 units/mg, bovine pancreas, Calbiochem), thermolysin (100 units/mg, bacterial, Sigma), subtilisin (15 units/mg, bacterial, Sigma); or aminopeptidase (50 units $/ \mathrm{mg}$, porcine intestinal mucosa, Sigma). These enzyme-containing aliquots were adjusted to $\mathrm{pH}$ 8 , incubated for $3 \mathrm{hr}$ at $37^{\circ} \mathrm{C}$, returned to $\mathrm{pH} 7$, and placed for $10 \mathrm{~min}$ in a boiling water bath to stop the reaction. Control samples were obtained by processing unconditioned HFRM through the same enzyme treatments, mixing with it untreated F10 fraction, and titrat- 
TABLE I

Analysis of trophic activities for various CNS and PNS test neurons in several conditioned media and ultrafiltration-derived fractions

Test neurons were dissociated from fetal rat hippocampus (rHIPP18), striatum (rSTR18), or septum (rSEPT18) and chick embryo forebrain (cFB8) for CNS sources; and from neonatal mouse dorsal root ganglia (mDRG) and chick embryo dorsal root ganglia (cDRG10), sympathetic (cSG11) or ciliary (cCG8) ganglia for PNS sources (end numbers indicate embryonic age). Conditioned media were collected from rat schwannoma (RCM), rat glioma (C6CM), mouse Schwann (SCM), rat cerebral astroglia (ACM), and chick embryo heart (HCM) cell cultures. They were tested in their native state ("crude CMs") or after processing on PM10 ultrafiltration membranes (R10 = retentates dialyzed and reconstituted to starting volume; F10 = filtrates, tested directly). F10 fractions were also processed through a UM2 membrane, and the corresponding filtrate fractions (F2) were collected and tested. Data were derived from titration curves over a 300 -fold dilution of the test samples. Values given are from one representative experinent. A replicate experiment gave results which agreed within $20 \%$.

\begin{tabular}{|c|c|c|c|c|c|c|c|c|}
\hline \multirow{3}{*}{ Trophic Sources } & \multicolumn{8}{|c|}{ Trophic Titers } \\
\hline & \multicolumn{4}{|c|}{ CNS Neurons } & \multicolumn{4}{|c|}{ PNS Neurons } \\
\hline & rHIPP18 & rSTR18 & rSEPT18 & $\mathrm{cFB8}$ & $\mathrm{mDRG}$ & cDRG10 & $\mathrm{cSG} 11$ & $\overline{\mathrm{cCG} 8}$ \\
\hline \multicolumn{9}{|l|}{ Crude CMs } \\
\hline $\mathrm{RCM}$ & 39 & 34 & 45 & 35 & 2.0 & 0.8 & 0 & 0 \\
\hline $\mathrm{C} 6 \mathrm{CM}$ & 38 & 32 & 42 & 29 & 3.0 & 0.7 & 2.4 & 1.0 \\
\hline HCM & 17 & 17 & 17 & 19 & 0 & 1.3 & 2.6 & 0 \\
\hline \multicolumn{9}{|l|}{ R10 (retentates) } \\
\hline RCM & 0 & 0 & 0 & 0 & 4.3 & 0.8 & 0.5 & 0 \\
\hline $\mathrm{C} 6 \mathrm{CM}$ & 0 & 0 & 0 & 0 & 3.0 & 0.6 & 1.9 & 1.1 \\
\hline SCM & 0 & 0 & 0 & 0 & 1.4 & 0.4 & 5.8 & 3.3 \\
\hline $\mathrm{ACM}$ & 0 & 0 & 0 & 0 & 0.7 & 1.5 & 2.3 & 1.5 \\
\hline SCM & 17 & 17 & 17 & 16 & 0 & 0 & 0 & 0 \\
\hline $\mathrm{ACM}$ & 7 & 6 & 8 & 8 & 0 & 0 & 0 & 0 \\
\hline $\mathrm{HCM}$ & 16 & 17 & 15 & 17 & 0 & 0 & 0 & 0 \\
\hline \multicolumn{9}{|l|}{ F2 (filtrates) } \\
\hline RCM & 37 & 33 & 37 & 35 & & & & \\
\hline $\mathrm{C} 6 \mathrm{CM}$ & 35 & 30 & 40 & 30 & & & & \\
\hline SCM & 15 & 17 & 19 & 15 & & & & \\
\hline $\mathrm{ACM}$ & 9 & 6 & 8 & 7 & & & & \\
\hline $\mathrm{HCM}$ & 15 & 16 & 15 & 17 & & & & \\
\hline
\end{tabular}

ing the activity of the mixture. No such processing of control samples led to decreased neuronal survival.

All of these physical or enzymatic treatments applied to the RCM-F10 material failed to affect its CNS activity (Table II). Thus, the RCM-derived low molecular weight trophic agent(s) addressing intrinsic CNS neurons is insensitive to heat, acid and alkaline $\mathrm{pHs}$, and to the action of various proteolytic and peptidolytic enzymes.

\section{Discussion}

In the present report, we have described a category of trophic agents promoting neuronal survival in vitro, which differ by at least two features from the more traditional neuronotrophic factors under study in this and other laboratories (cf. Varon and Adler, 1981):

1. The new trophic agents are necessary for the shortterm survival of CNS neurons in dissociated cell cultures but are unable by themselves to support the survival of PNS neurons. CNS cells cultured in the absence of the low molecular weight agent for $24 \mathrm{hr}$ show numerous cell ghosts, indicating that the neurons were able to attach without the trophic agent, but they were unable to survive. Cell-cell as well as cell-substratum interactions may be important in the action of a neuronotrophic agent. Thus, "attachment" factors and "trophic" factors cannot
TABLE II

Effect of various treatments on CNS trophic activity in RN22 conditioned medium ultrafiltrate

An ultrafiltrate from RN22 conditioned medium was prepared using a PM10 membrane. CNS trophic activity was examined using neurons from fetal rat hippocampus (rHIPP18), striatum (rSTR18), or septum (rSEPT18) and chick embryo forebrain (cFB8). The different treatment modalities and cell preparations are described in the text. Values are averaged from two indendent titration curves.

\begin{tabular}{lcccc}
\hline \multirow{2}{*}{ Treatment } & \multicolumn{4}{c}{$\%$ Control Titer } \\
\cline { 2 - 5 } & rHIPP18 & rSTR18 & rSEPT18 & CFB8 \\
\hline None & 100 & 100 & 100 & 100 \\
$100^{\circ} \mathrm{C}(30 \mathrm{~min})$ & 100 & 100 & 100 & 100 \\
$\mathrm{pH} 4(3 \mathrm{hr})$ & 100 & 100 & 100 & 100 \\
pH $10(3 \mathrm{hr})$ & 100 & 100 & 100 & 100 \\
Trypsin & 100 & 100 & 100 & 100 \\
$\alpha$-Chymotrypsin & 100 & 100 & 100 & 100 \\
Thermolysin & 100 & 100 & 100 & 100 \\
Protease & 100 & 100 & 100 & 100 \\
Aminopeptidase & 100 & 100 & 100 & 100 \\
\hline
\end{tabular}

be distinguished from one another until full knowledge of their mechanisms will be achieved. Long-term survival (beyond 1 week) requirements by these CNS neuronal cultures remain to be investigated. 
2. The CNS neurons responsive to these agents were derived from various parts of the brain (Table I) as well as from spinal cord (Manthorpe et al., 1982) and from both rodent and avian sources, suggesting that the trophic role of these agents, while restricted to CNS neurons, may not be selective for individual neuronal subsets.

3. The new trophic activity resides with low molecular weight (LMW) agents $\left(M_{\mathrm{r}}<1000\right)$ rather than with protein macromolecules. The chemical nature of the new agents remains to be ascertained. It is possible that the LMW agents are nutrients (e.g., amino acids) or intermediate metabolites specifically and critically required for the survival of CNS neurons. Alternatively, they may prove to be hormones or peptides with a hitherto unrecognized trophic role for such neurons. In either case, their selective relevance for CNS neurons amply justifies further efforts to identify their molecular nature and to isolate them from the other LMW constituents of their source-conditioned media.

The recognition of this new category of LMW trophic agents opens up interesting new lines of investigation besides their identification and isolation. Their presence in a variety of glia-conditioned media (as well as other $\mathrm{CMs}$ ) provides opportunities to study regulatory mechanisms concerned with their production and release, as well as to explore additional modes by which neurons and glial cells may interact with one another (cf. Varon and Somjen, 1979). Investigation of the access by the LMW agents to the target neurons (e.g., surface binding, internalization by transmembrane transport or endocytosis) and of the molecular mechanisms through which they exert their effects will draw attention to cellular and metabolic processes which are especially critical for the survival of CNS, but not PNS, neurons. Lastly, in vitro survival of CNS neurons can also be supported by protein-like neuronotrophic factors, present in fluids collected in vivo from lesions imparted to either peripheral (Longo et al., 1983) or central (Manthorpe et al., 1983) neural tissues or extractable from brain tissues (Turner et al., 1982; I. Selak, G. Barbin, M. Manthorpe, and S. Varon, manuscript in preparation). Although it is likely that such macromolecular trophic factors operate via mechanisms different from those used by the LMW agents, one will have to ascertain whether the two types of trophic agents may have additive effects (at the overall population level) or cooperative ones (at the level of shared target neurons).

\section{References}

Adler, R., and S. Varon (1980) Cholinergic neuronotrophic factors. V. Segregation of survival- and neurite-promoting activities in heart conditioned media. Brain Res. 188: 437448.

Banker, G. (1980) Trophic interactions between astroglial cells and hippocampal neurons in culture. Science 209: 809-810.

Barbin, G., I. Selak, M. Manthorpe, and S. Varon (1984) Use of CNS neuronal cultures for the detection of neuronotrophic agents. Neuroscience, in press.

Barde, Y. A., R. M. Lindsay, D. Monard, and H. Thoenen (1978) New factor released by cultured glioma cells supporting survival and growth of sensory neurones. Nature (Lond.) 274: 818 .

Helfand, S. L., R. J. Riopelle, and N. K. Wessells (1978) Nonequivalence of conditioned medium and nerve growth factor for sympathetic, parasympathetic, and sensory neurons. Exp. Cell Res. 113: 39-45.

Longo, F. M., M. Manthorpe, and S. Varon (1982) Spinal cord neuronotrophic factors (SCNTFs). I. Bioassay of schwannoma and other conditioned media. Dev. Brain Res. 3: 277294.

Longo, F. M., M. Manthorpe, S. D. Skaper, G. Lundborg, and S. Varon (1983) Neuronotrophic activities accumulate in vivo within silicone nerve regeneration chambers. Brain Res. 261: 109-117.

Manthorpe, M., S. Varon, and R. Adler (1981) Neurite-promoting factor (NPF) in conditioned medium from RN22 schwannoma cultures: Bioassay, fractionation and other properties. J. Neurochem. 37: 759-767.

Manthorpe, M., F. M. Longo, and S. Varon (1982) Comparative features of spinal neuronotrophic factors in fluids collected in vitro and in vivo. J. Neurosci. Res. 8: 241-250.

Manthorpe, M., M. Nieto-Sampedro, S. D. Skaper, E. R. Lewis, G. Barbin, F. M. Longo, C. W. Cotman, and S. Varon (1983) Neuronotrophic activity in brain wounds in the developing rat. Correlation with implant survival in the wound cavity. Brain Res. 267: 47-56.

Mirsky, R., L. M. B. Wendon, P. Black, C. Stolkin, and D. Blay (1978) Tetanus toxin: A cell surface marker for neurones in culture. Brain Res. 148: 251-259.

Müller, H. W., and W. Seifert (1982) A neuronotrophic factor (NTF) released from primary glial cultures supports survival and fiber outgrowth of cultured hippocampal neurons. J. Neurosci. Res. 8: 195-204.

Skaper, S. D., R. Adler, and S. Varon (1979) A procedure for purifying neuron-like cells in cultures from central nervous tissues with a defined medium. Dev. Neurosci. 2: 233-237.

Turner, J. E., Y. A. Barde, M. Schwab, and H. Thoenen (1982) Extract from brain stimulates neurite outgrowth from fetal rat retinal explants. Dev. Brain Res. 6: 77-84.

Varon, S., and R. Adler (1980) Nerve growth factors and control of nerve growth. Curr. Top. Dev. Biol. 16:207-252.

Varon, S., and R. Adler (1981) Trophic and specifying factors directed to neuronal cells. Adv. Cell. Neurobiol. 2: 115-163.

Varon, S., and G. Somjen (1979) Neuron-glia interactions. Neurosci. Res. Program Bull. 17: 1-239.

Varon, S., S. D. Skaper, and M. Manthorpe (1981) 'Trophic activities for dorsal root and sympathetic ganglionic neurons in media conditioned by Schwann and other peripheral cells. Dev. Brain Res. 1: 73-87. 